\title{
Genital tuberculosis in a tamoxifen-treated postmenopausal woman with breast cancer and bloody vaginal discharge Ioannis Neonakis' ${ }^{1}$, Elpis Mantadakis², Zoe Gitti1 ${ }^{1}$, Ioanna Mitrouska3 , Louis George Manidakis ${ }^{4}$, Sofia Maraki ${ }^{1}$ and George Samonis*2
}

\begin{abstract}
Address: ${ }^{1}$ Laboratory of Mycobacteriology, Department of Clinical Bacteriology, Parasitology, Zoonoses, and Geographical Medicine, University Hospital of Heraklion, 71201 Heraklion, Crete, Greece, 2Department of Internal Medicine, University Hospital of Heraklion, 71201 Heraklion, Crete, Greece, ${ }^{3}$ Department of Thoracic Medicine, University Hospital of Heraklion, 71201 Heraklion, Crete, Greece and ${ }^{4}$ Department of Obstetrics and Gynecology, University Hospital of Heraklion, Heraklion, Crete, Greece

Email: Ioannis Neonakis - neos@med.uoc.gr; Elpis Mantadakis - elpis.mantadakis@gmail.com; Zoe Gitti - zgitti@in.gr; Ioanna Mitrouska - mitrouska@med.uoc.gr; Louis George Manidakis - manidakislg@hotmail.com; Sofia Maraki - sofiamaraki@in.gr; George Samonis* - georgsec@med.uoc.gr

* Corresponding author
\end{abstract}

\section{Published: 0 I September 2006}

Annals of Clinical Microbiology and Antimicrobials 2006, 5:20 doi:I0.I I86/I476-07II-520

This article is available from: http://www.ann-clinmicrob.com/content/5/I/20

(c) 2006 Neonakis et al; licensee BioMed Central Ltd.

This is an Open Access article distributed under the terms of the Creative Commons Attribution License (http://creativecommons.org/licenses/by/2.0), which permits unrestricted use, distribution, and reproduction in any medium, provided the original work is properly cited.
Received: 05 July 2006

Accepted: 01 September 2006

\begin{abstract}
Background: Female genital tuberculosis is an uncommon disease that is rarely diagnosed in developed countries.

Case presentation: A 61-year-old postmenopausal woman who had undergone surgery and treated with adjuvant chemotherapy for infiltrating ductal carcinoma of the breast five years ago, presented with bloody vaginal discharge, fatigue, weight loss, and low grade fevers at night for two months. Histological examination of the endometrium, done based on the suspicion of a second primary cancer due to the tamoxifen therapy, revealed a granulomatous reaction. Liquid and solid mycobacterial cultures of the tissues were performed. Although the acid fast staining was negative, the liquid culture was positive for Mycobacterium tuberculosis. Involvement of other systems was not detected. The patient was treated with a three-drug antituberculosis regimen for 9 months and recovered fully.

Conclusion: Female genital tuberculosis is a rare but curable disease that should be included in the differential diagnosis of women with menstrual problems. Early diagnosis is important and may prevent unnecessary invasive procedures for the patient.
\end{abstract}

\section{Background}

Tuberculosis (TB) predominately presents with pulmonary disease, although extra-pulmonary TB is not uncommon [1-4]. Diagnosis of extra-pulmonary TB is often difficult, because of its non-specific clinical and laboratory findings [2]. Female genital TB (FGTB) is usually a symptom-less disease diagnosed during investigations for infertility [5-7]. Symptomatic disease usually presents with pelvic pain or menstrual irregularities $[8,9]$. FGTB is extremely rare in postmenopausal women [10]. We present a case of FGTB in a tamoxifen-treated postmenopausal woman with breast cancer and bloody vaginal discharge. 


\section{Case presentation}

A 61-year-old postmenopausal woman diagnosed in the past with breast cancer presented to her Oncologist for regular follow up. The patient complained of fatigue, weight loss, persistent slight evening pyrexia, a mild lower abdominal pain for the last two months, along with leukorrhea and bloody vaginal discharge for the last three weeks. Five years ago, during the perimenopausal period, she was diagnosed with an infiltrating ductal carcinoma of the breast. She underwent lumpectomy followed by adjuvant chemotherapy and radiotherapy. Since then she was receiving oral tamoxifen that she was due to stop in one month and had no clinical, laboratory or radiological evidence of recurrent or metastatic disease.

Physical examination revealed mild abdominal distension and an enlarged, tense, and tender uterus. A chest radiograph was negative, while a full blood count, serum electrolytes, and liver function tests were normal. A transvaginal ultrasonogram showed an enlarged uterus with a $7 \mathrm{~mm}$ endometrial thickness (normal for age up to 4 $\mathrm{mm}$ ). Hysteroscopy and endometrial aspiration biopsy performed because of the suspicion of a second primary cancer of the endometrium from the tamoxifen therapy disclosed necrotic debris with an extensive granulomatous reaction, numerous tubercles composed of epithelioid cells, and multinucleated giant cells with no central necrosis. These findings raised the suspicion of FGTB. Hence, samples from the endocervix and the endometrium were obtained with the use of a sterile Cyto Brush for PAP smear, suspended in sterile water, and processed according to standard guidelines [11] followed by cultures in solid (Lowenstein-Jensen, BioMerieux, France) and liquid media (BacT/Alert 3D, Organon Teknika, NC). Although acid-fast staining was negative, the liquid culture gave a positive signal after 7 days of incubation. The presence of Mycobacterium tuberculosis was verified by AccuProbe assay (Gen-Probe, CA) and phenotypic-biochemical analysis. Since the samples submitted for culture from the endocervix and the endometrium were not separated, it is unclear if TB was limited to the endocervix, the endometrium or both.

Tuberculin skin testing was positive $(>15 \mathrm{~mm})$, while detailed family history revealed that the patient's mother and sister had died 30 and 10 years ago from TB. The patient had taken close care of both. No other family members had symptoms suspicious of TB. Interestingly, the patient had refused this family history at presentation to her Oncologist. Finally, she admitted the contact history to patients infected with TB only after the results of the cultures were available. In order to rule out pulmonary and renal spread of the disease, imaging and laboratory evaluation with chest radiograph and examination of multiple early morning sputum and urine samples with acid-fast staining and cultures was performed and showed no lung or renal involvement.

Drug susceptibility testing was performed by the proportional methods on Lowenstein-Jensen medium. The isolated strain was resistant to pyrazinamide $(200 \mu \mathrm{g} / \mathrm{ml})$, streptomycin $(4 \mu \mathrm{g} / \mathrm{ml})$, ethionamide $(30 \mu \mathrm{g} / \mathrm{ml})$, rifampicin $(20 \mu \mathrm{g} / \mathrm{ml})$ and pefloxacine $(25 \mu \mathrm{g} / \mathrm{ml})$ and susceptible to isoniazid $(0.2 \mu \mathrm{g} / \mathrm{ml})$, rifampicin $(40 \mu \mathrm{g} /$ $\mathrm{ml})$, ethambutol $(2 \mu \mathrm{g} / \mathrm{ml})$, streptomycin $(10 \mu \mathrm{g} / \mathrm{ml})$ and para-4-aminosalicylic acid $(0.5 \mu \mathrm{g} / \mathrm{ml})$. The patient was treated with a three-drug regimen of isoniazid $(300 \mathrm{mg} /$ day), rifampicin (600 $\mathrm{mg} /$ day) and ethambutol (1500 $\mathrm{mg} /$ day) for nine months. Within a month after the beginning of antituberculosis therapy, the vaginal discharge disappeared along with resolution of the fatigue, a two kilogram weight gain, and complete resolution of the persistent evening pyrexia and the lower abdominal pain.

\section{Discussion}

Despite the remarkable efforts to control TB worldwide over the past decades, almost two million people still die of this disease every year, justifying the declaration of TB as a public health emergency by the $44^{\text {th }}$ World Health Assembly. FGTB is an uncommon presentation. Its exact incidence remains unknown, as the majority of the cases remain undiagnosed. In Western countries the incidence of FGTB is estimated to be $<1 \%$, whereas in some African and Asian countries it reaches 15-19\% [12,13].

Although in some cases cervical TB is thought to be the primary infection $[14,15]$, FGTB is usually a secondary complication of pulmonary TB. In most cases it begins with a focus in the endosalpinx and spreads to the endometrium, the ovaries, occasionally to the cervix and rarely the vagina [8]. In our case it is unclear whether the disease was limited to the endocervix and/or the endometrium since the samples provided for culture were not separated.

In the developing world, almost nine out of ten cases are young women between 20 and 40 years of age [16], whereas, in Western countries it is usually diagnosed in women over the age of 40, usually pre-menopausal ones [17-19]. The atrophic endometrium of postmenopausal women is thought to offer a poor milieu for the growth of M. tuberculosis [10]. Hence, in contrast to premenopausal women where infertility is the main symptom leading the suspected gynecologist to seek for FGTB, in postmenopausal women vaginal bleeding is the usual presenting symptom of the disease.

FGTB is rarely diagnosed in developed countries. Symptoms are usually absent and there is often no evidence of $\mathrm{TB}$ in other organs. If present, the symptoms are mild and 
local, such as abdominal pain or menstrual irregularities. Infertility is the most common consequence [7]. Due to its rarity and its mild clinical picture, the index of suspicion for the diagnosis of FGTB among gynecologists is usually low. This is unfortunate since early detection and proper treatment can, in some cases, prevent irreversible damage to the fallopian tubes and the endometrium. Once fibrosis is established, fertility is generally difficult to restore even with appropriate treatment [9]. Nevertheless, early diagnosis can save the patient from a series of invasive diagnostic or therapeutic procedures. To this end, identification of high risk patients is helpful. Suspicions should be raised if a woman has a personal history of latent tuberculous infection or previous contact with persons suffering from TB. In the present case, persistent questioning revealed the patient's prior close contact with infected persons. Furthermore, the clinician should be suspicious if the patient originates from areas where TB or AIDS are endemic, since more than $3 \%$ of new cases of $\mathrm{TB}$ are related to HIV infection [13]. Additionally, in some developing countries, as much as $60-90 \%$ of extrapulmonary TB occurs in HIV positive patients $[13,20]$. Since genital disease often causes no physical symptoms, infertile women should be examined for $\mathrm{TB}$, especially in case of predisposing factors. This is also true for post-menopausal women with vaginal bleeding. Samples from the endometrium and the endocervix should be submitted for cytological examination, acid fast stains, and cultures, since combination of different methods is likely to have a higher diagnostic yield for TB.

The diagnostic role of a positive Mantoux (purified protein derivative-PPD) skin test is controversial. In a recent study, almost $45 \%$ of infertile women with strong indirect evidence of pelvic TB, such as laparoscopic findings (thickened tubes, areas of caseation, etc.), had a negative PPD skin test [21]. On the other hand, in a group of 27 infertile women with a positive PPD skin test, only 11 had clear laparoscopic findings suggestive of FGTB. The other conventional diagnostic methods of $\mathrm{TB}$, such as chest radiographs, urine, and sputum cultures are not specific for FGTB, but are helpful to rule out dissemination to other organs. Infertile women with a positive PPD skin test should undergo early laparoscopy for direct visualization of the fallopian tubes and for collection of material from the pelvis for pathological examination and cultures [21]. Laparoscopic findings, such as the presence of excessive peritoneal fluid, rigid and almost immobile tubes, presence of tubercles or caseous material are indicative but not pathognomonic of FGTB. The value of other diagnostic means, such as sonography or hysterosalpingography remains controversial [19].

Histological detection of typical granulomata is sufficient for diagnosis of pelvic TB, providing that all other causes of granulomatous reactions have been ruled out. Differential diagnosis includes tularemia, sarcoidosis, brucellosis, amoebiasis, schistosomiasis and foreign body reactions $[9,22]$. Identification of extragenital TB strongly supports the diagnosis. Nevertheless, the gold standard for diagnosis of FGTB is the isolation of M. tuberculosis from appropriate specimens, usually biopsy materials from the endometrium. Samples can be used for the detection of M. tuberculosis DNA by PCR or can be cultured [16]. Efforts are currently made to use menstrual blood, when available, for molecular or serological diagnosis [16].

In the present case, the differential diagnosis included a second primary cancer of the endometrium due to the prolonged use of tamoxifen and possible genital TB or other granulomatous diseases. The use of a Cyto Brush was effective for proper sample taking, while isolation of M. tuberculosis was achieved by conventional methods. The use of Cyto Brush has some advantages compared with biopsy, such as simplicity, ease of use, low cost, and minimal patient discomfort. To our knowledge, no studies have directly compared biopsy versus Cyto Brush or Cyto Brush versus other conventional sampling methods, e.g., wooden spatulas or cotton swabs for isolation of mycobacteria, although the hydrophobic nature of the lipid-containing cell wall of mycobacteria inhibits their transfer from the cotton fibers to the aqueous culture medium [23].

Acid-fast staining was negative in our patient. This test is infrequently positive, since approximately $10^{5}$ mycobacteria per milliliter are required in order to have a positive result [19]. On the other hand, liquid mycobacterial cultures are useful, usually leading to isolation within days. Nowadays, a series of automatic liquid culture systems are available contributing to faster diagnosis.

In conclusion, FGTB is a rare but curable disease that should be included in the differential diagnosis of postmenopausal vaginal bleeding. Early diagnosis is important and it can save the patient from unnecessary invasive procedures.

\section{Abbreviations}

TB: tuberculosis, FGTB: Female genital tuberculosis, PPD: purified protein derivative

\section{Competing interests}

The author(s) declare that they have no competing interests.

\section{Authors' contributions}

IN carried out the laboratory procedures and drafted the paper. EM helped to draft the manuscript and revised it critically. ZG participated in carrying out the laboratory 
procedures. IM handled the patient's antituberculosis treatment. LGM performed the hysteroscopy and endometrial aspiration biopsy of the patient. SM participated in the coordination of the clinical and laboratory work-up. GS conceived the report, treated the patient for the breast cancer, revised the manuscript and gave final approval of the submitted version. All authors have read and approved the final manuscript.

\section{Acknowledgements}

Written consent was obtained from the patient for publication of the study.

\section{References}

I. Gonzalez OY, Adams G, Teeter LD, Bui TT, Musser JM, Graviss EA: Extra-pulmonary manifestations in a large metropolitan area with a low incidence of tuberculosis. Int J Tuberc Lung Dis 2003, 7: I I78-II85.

2. Rasolofo RV, Menard D, Auregan G, Gicquel B, Chanteau S: Extrapulmonary and pulmonary tuberculosis in Antananarivo (Madagascar): high clustering rate in female patients. J Clin Microbiol 2002, 40:3964-3969.

3. Harries AD, Hargreaves NJ, Kwanjana JH, Salaniponi FM: The diagnosis of extrapulmonary tuberculosis in Malawi. Trop Doct 2003, 33:7-II.

4. Lewis KE, Stephens C, Shahidi MM, Packe G: Delay in starting treatment for tuberculosis in east London. Commun Dis Public Health 2003, 6:133-138.

5. Goldin AG, Baker WT: Tuberculosis of the female genital tract. J Ky Med Assoc 1985, 83:75-76.

6. Margolis K, Wranz PA, Kruger TF, Joubert J], Odendaal HJ: Genital tuberculosis at Tygerberg Hospital - prevalence, clinical presentation and diagnosis. S Afr Med J 1992, 81 : I2-15.

7. Namavar Jahromi B, Parsanezhad ME, Ghane-Shirazi R: Female genital tuberculosis and infertility. Int J Gynaecol Obstet 200I, 75:269-272

8. Haas DW, Des Prez RM: Mycobacterium Tuberculosis. In Principles and Practice of Infectious Diseases Edited by: Mandell GL, Bennett JE, Dolin R. Philadelphia, Churchill Livingstone; 1995:2238.

9. Lamba H, Byrne M, Goldin R, Jenkins C: Tuberculosis of the cervix: case presentation and a review of the literature. Sex Transm Infect 2002, 78:62-63.

10. Maestre MA, Manzano CD, Lopez RM: Postmenopausal endometrial tuberculosis. Int J Gynaecol Obstet 2004, 86:405-406.

II. Kubica GP, Gross WM, Hawkins JE, Sommers HM, Vestal AL, Wayne LG: Laboratory services for mycobacterial diseases. Am Rev Respir Dis 1975, I I 2:773-787.

12. Punnonen R, Kiilholma P, Meurman L: Female genital tuberculosis and consequent infertility. Int J Fertil 1983, 28:235-238.

13. Giannacopoulos KCh, Hatzidaki EG, Papanicolaou NC, Relakis KJ, Kokori HG, Giannacopoulou CC: Genital tuberculosis in a HIV infected woman: a case report. Eur J Obstet Gynecol Reprod Biol 1998, 80:227-229.

14. Sutherland AM, Glen ES, MacFarlane JR: Transmission of genitourinary tuberculosis. Health Bull (Edinb) 1982, 40:87-91.

15. Richards MJ, Angus D: Possible sexual transmission of genitourinary tuberculosis. Int J Tuberc Lung Dis 1998, 2:439.

16. Abebe M, Lakew M, Kidane D, Lakew Z, Kiros K, Harboe M: Female genital tuberculosis in Ethiopia. Int J Gynaecol Obstet 2004, 84:24I-246.

17. Falk V, Ludviksson K, Agren G: Genital tuberculosis in women. Analysis of 187 newly diagnosed cases from 47 Swedish hospitals during the ten-year period 1968 to 1977. Am J Obstet Gynecol 1980, 138:974-947.

18. Manidakis LG, Angelakis E, Sifakis S, Stefanaki P, Kalogeraki A, Manidaki A, Koumantakis E: Genital tuberculosis can present as disseminated ovarian carcinoma with ascites and raised $\mathrm{Ca}$ I 25: a case report. Gynecol Obstet Invest 200I, 5 I:277-279.

19. Chow TW, Lim BK, Vallipuram S: The masquerades of female pelvic tuberculosis: case reports and review of literature on clinical presentations and diagnosis. J Obstet Gynaecol Res 2002, 28:203-210.
20. Narain JP, Raviglione MC, Kochi A: HIV-associated tuberculosis in developing countries: epidemiology and strategies for prevention. Tuber Lung Dis 1992, 73:3 II-32I.

21. Raut VS, Mahashur AA, Sheth SS: The Mantoux test in the diagnosis of genital tuberculosis in women. Int J Gynaecol Obstet 200I, 72:165-169.

22. Koller AB: Granulomatous lesions of the cervix uteri in Black patients. S Afr Med J 1975, 49:1228-32.

23. Koneman E, Allen S, Janda W, Schreckenberger P, Winn W: Mycobacteria. In Colour atlas and textbook of Diagnostic Microbiology Edited by: Koneman E, Allen S, Janda W, Schreckenberger P, Winn W. Philadelphia, Lippincott; 1997:898.
Publish with Biomed Central and every scientist can read your work free of charge

"BioMed Central will be the most significant development for disseminating the results of biomedical research in our lifetime. "

Sir Paul Nurse, Cancer Research UK

Your research papers will be:

- available free of charge to the entire biomedical community

- peer reviewed and published immediately upon acceptance

- cited in PubMed and archived on PubMed Central

- yours - you keep the copyright 